\title{
Awake perception is associated with dedicated neuronal assemblies in cerebral cortex
}

Authors: Anton Filipchuk ${ }^{1 *}$, Alain Destexhe ${ }^{*} \dagger$, Brice Bathellier ${ }^{1,2, * \dagger}$

\section{Affiliations:}

${ }^{1}$ Department for Integrative and Computational Neuroscience (ICN),Paris-Saclay Institute of Neuroscience (NeuroPSI), UMR9197 CNRS/University Paris Sud, CNRS, Building 32/33, 1 avenue de la Terrasse, 91190 Gif-sur-Yvette, France

2 Institut Pasteur, INSERM, Institut de l'Audition, 63 rue de Charenton, F-75012 Paris, France.

*Corresponding author. Email: brice.bathellier@cnrs.fr, alain.destexhe@,cnrs.fr, anton.filipchuk@,cnrs.fr

$\uparrow$ These authors contributed equally to this work

\begin{abstract}
Neural activity in sensory cortex combines stimulus responses and ongoing activity, but it remains unclear whether they reflect the same underlying dynamics or separate processes. Here we show that during wakefulness, the neuronal assemblies evoked by sounds in the auditory cortex and thalamus are specific to the stimulus and distinct from the assemblies observed in ongoing activity. In contrast, during anesthesia, evoked assemblies are indistinguishable from ongoing assemblies in cortex, while they remain distinct in the thalamus. A strong remapping of sensory responses accompanies this dynamical state change produced by anesthesia. Together, these results show that the awake cortex engages dedicated neuronal assemblies in response to sensory inputs, which we suggest is a network correlate of sensory perception.
\end{abstract}

One-Sentence Summary: Sensory responses in the awake cortex engage specific neuronal assemblies that disappear under anesthesia. 


\section{Main Text:}

It has long been noticed that the circuits of sensory areas in the cerebral cortex display intense ongoing activity in the absence of stimuli from their dedicated sensory modality (1). The role of this ongoing activity and its relationship to evoked sensory responses remains unclear. Initial observations made under anesthesia in the visual cortex of several mammals (2-4) have shown a striking similarity between ongoing activity patterns on the mesoscopic scale and sensory responses, suggesting that ongoing activity could be a form of replay of sensory responses. Similar results have been obtained in the rat primary auditory cortex (5).

However, recently, recordings in the visual cortex of awake mice have shown that ongoing cortical activity in wakefulness is highly correlated to changes in the arousal $(6,7)$ and the facial motor activity pattern of the animal $(8,9)$. Arousal related fluctuations are also seen in the visual thalamus (10). Although the direction of causality between behavioral and cortical observables remains to be established, this suggests that ongoing cortical dynamics in the awake state is more than a replay of past sensory activity. In line with this, it was also observed that even if ongoing and evoked activity recruit similar sets of neurons, they correspond to activity patterns that live in orthogonal neuronal dimensions $(8,11)$. These conflicting observations across physiological states suggest that anesthesia triggers a profound transformation of neuronal dynamics in the cortical circuits and eventually beyond. However, the lack of data allowing direct comparison of thalamo-cortical activity in the same neurons across anesthesia and wakefulness hinders the resolution of this question, which is all the more important that sensory representations have been alternatively studied in both states.

Taking advantage of the robustness of GCAMP6s-based (12) two-photon calcium imaging for the assignment of neuronal activity to identified neurons, we contrasted neural population activity in the auditory cortex of head-fixed mice, in the awake state (Fig. 1A) and during light isoflurane anesthesia (Fig. 1B). A first 10 min imaging session without auditory stimuli was followed by a 15 min session in which we presented 50 different simple and complex sounds (500ms, Fig. S1), novel for the animal, each repeated 12 times and delivered in a random order. After sound presentation, another session without auditory stimuli was performed to evaluate the impact of sound presentations on ongoing activity. Then, the same protocol was repeated under anesthesia. $728+/-180$ (474-955) neurons could be imaged simultaneously in cortical layer 2/3 over a 1x1 mm field of view covering about one quarter of the entire auditory cortex. Automated segmentation (13), fluorescence trace extraction and spike time estimation was performed using the MLSpike algorithm (14)(Fig. S1), yielding population activity rasters for well-identified neurons across sessions and physiological states (Fig. 1C-D, S1).

In line with previous observations $(5,15-17)$, inspection of the raster plots and of the instantaneous population firing curves revealed short synchronous population activity events, which occurred both in the anesthetized and awake states but seemed more contrasted and stereotypical under anesthesia (Fig. 1C-D, S1). We extracted these events by applying a baseline corrected (18) threshold to the population firing rate above which a synchronous event could not be explained by the fluctuations of summed independent Poisson processes (Fig. 1C-D, S1). Population events were short but of variable durations $(327+/-131 \mathrm{~ms}, \mathrm{n}=11$ mice in awake state, $414+/-237 \mathrm{~ms}, \mathrm{n}=6$ mice under anesthesia) and appeared both during the stimulation-free 
$(0.42+/-0.1 \mathrm{~Hz}, \mathrm{n}=11$ mice in awake state, $0.51+/-0.11 \mathrm{~Hz}, \mathrm{n}=6$ mice under anesthesia) and sound delivery protocols (Fig. 1C). Relying on the same detection criterion, the probability that a population event appeared just after a sound onset or offset was $52+/-20 \%$, ( $\mathrm{n}=11$ mice) in the awake state and $41+/-8 \%$ ( $n=6$ mice) under anesthesia, with a large disparity across sounds. Some sounds drove detectable population events on almost every trial, others did not (Fig. S1).

We then asked if events observed in the absence of stimuli resemble the activity generated by sensory stimuli, and in particular if similar assemblies of neurons are recruited in both cases. For on-going activity, we identified each detected population event to the assembly of neurons that fired at least one putative action potential during the event. To avoid unnecessary thresholding effects, for sound-evoked activity, the assembly of responsive neurons was the neurons that fired at least one putative action potential during sound presentation. Similarity between assemblies was measured based on the correlation between binary population vectors (entry 1 for neurons belonging to the assembly and 0 otherwise), which had the length of the entire neuronal population. Then we used a simple approach to account for the intrinsic variability of neural responses and noise introduced by spike estimation errors (14). We performed hierarchical clustering to organize all ongoing assemblies in groups of similar patterns and displayed the matrix of pairwise similarity between individual ongoing assemblies and single trial responses to all tested sounds (Fig. S1, Fig. 1E,F). Visual inspection of sample matrices for pairs of sounds and ongoing assembly clusters indicated overall a low similarity between ongoing assemblies and evoked sound responses in the awake state contrasting with high similarity levels in anesthesia in the same neuronal population (Fig. 1E,F). To quantify this, we measured, for each sound and imaging session, the mean similarity between individual evoked responses and the assemblies of the most similar ongoing assembly cluster. Plotting this measure against the reproducibility of evoked responses across renditions and of the assemblies within a cluster, it became evident that similarity was below reproducibility levels in the awake state whether ongoing assemblies were taken before (Fig. 1G) or after (Fig. 1H) sound presentations. This result was robust to changes in clustering parameters (Fig. S2), and cortical depth (Fig. S3). It also held for assemblies observed in between sound stimulations (Fig. S4), and when on-going assemblies were compared to sound responses clustered in the same way (Fig. S2). Hence, evoked responses and ongoing assemblies correspond to distinct population activity patterns in awake freely listening mice.

However, in anesthesia a profound reorganization of cortical dynamics was observed. First, assemblies and sound responses seen under anesthesia were clearly distinct from those seen in the awake state (Figs. 1I-J, S5). This indicates that a large fraction of neurons change their apparent stimulus tuning under anesthesia (Fig. S6). Interestingly, some response types were preserved under anesthesia, suggesting the existence of more robust response modes (Fig. S6). Second, we observed that evoked responses and ongoing assemblies are identical under anesthesia, as measured through their equal similarity and reproducibility levels (Figs. 1K-L, S5). This suggests that assemblies in on-going activity and in sensory responses emerge from a stereotypical process in anesthesia, while in the awake state, auditory cortex develops a much richer repertoire of activity patterns which carries more information, in particular about sounds. Corroborating this, we observed that assemblies are much less diverse under anesthesia than in 
wakefulness (Fig. 1F) and that sound decoding performance based on cortical activity is much larger in wakefulness than under anesthesia (Fig. S7).

To evaluate the contribution of thalamic inputs in the reorganization of cortical activity, we imaged thalamic boutons in the auditory cortex labeled with GCamP6s through stereotaxic AAV virus injections in the primary and secondary auditory thalamus (Fig. 2A) (19, 20). Signals from individual thalamic axons and boutons were extracted using the same automated methods as for somatic fluorescence, applied to a smaller field of view in layer 1 of the auditory cortex. Using the MLSpike algorithm we obtained estimates of the spike trains underlying thalamo-cortical synaptic release in cortex (Fig. 2B-C). As in the cortex, population activity in the thalamus displayed short synchronous population events that were more contrasted under anesthesia (Fig. 2B-C). Measuring population vector correlation as previously (Fig. 2D,E), we observed that ongoing assemblies were significantly different from evoked activity in the awake state in the thalamic output as in the cortex (Fig. 2F,G). Thus, the thalamic input provides separate information streams based on which the cortex can build distinct assemblies in its ongoing and evoked activity.

Interestingly, under anesthesia, neural assemblies in the thalamic input were also profoundly reshaped as compared to assemblies seen in the awake state (Fig. 2H, I). This involved changes in the tuning of individual neurons to sounds, parallel to those observed in the cortex (Fig. S6). Ongoing activity patterns in thalamic fiber under anesthesia (Fig. 2G) were often observed to be distinct before and after sound presentation, which may reflect unavoidable changes in the state of anesthesia. However, unlike what we observed in cortical neurons, ongoing assemblies observed before or after sound presentation in thalamic fibers activity were not identical to evoked activity patterns (Fig. 2J, K). Therefore, during anesthesia, the thalamus sends sensory driven and ongoing activity inputs that are different, but cortical activity does not take in account this difference.

To find further evidence for this observation, we analyzed single neuron properties in cortical activity, seeking to identify distinctive functional markers of ongoing and evoked activity. We measured for each neuron the probability to emit at least one putative action potential during a spontaneous assembly and during presentation of a sound (500 $\mathrm{ms}$ after onset). In the awake state, the scattering of probabilities was broad (Fig. 3A). Substantial fractions of neurons were more specific to ongoing assemblies $(\sim 1 / 6)$ or more specific to sound responses $(\sim 1 / 6$, Fig. 3A). Remaining neurons displayed similar probabilities to participate in ongoing assemblies or evoked response $(\sim 2 / 3$, Fig. 3A). Specific neurons were large contributors to the identity and robustness of on-going assemblies and sound responses. Indeed, if specific neurons were removed by retaining neurons for which the absolute difference between ongoing and evoked probabilities was below one mean absolute deviation of the full distribution, population vector reproducibility levels drastically dropped both for ongoing assemblies and sound responses (Fig. 3B), although some information about sounds remained (Fig. S7). Corroborating this, when nonspecific neurons were discarded, population vector reproducibility levels were boosted (Fig. 3CE). Also, if only spontaneous assembly neurons were retained, reproducible and sound-specific sound response patterns disappeared (Fig. 3D), leading to a severe drop in sound decoding (Fig. S7). This indicates that even if no neuron is fully specific of on-going or evoked activity as 
previously observed in the visual cortex (8), small and distinct neuronal subpopulations are specialized in carrying sound specific information versus forming different on-going activity motifs.

Tracking non-specific, sound specific and ongoing assembly specific subpopulations during anesthesia further uncovered the profound transformation of cortical dynamics. Plotting the probability to fire in an ongoing assembly against the probability to fire in a sound-evoked response indicated that the two specific neuronal subpopulations vanished under anesthesia and were merged to a large non-specific subpopulation (Fig. 3F, S8). This reshuffling of cellular properties could also be seen by plotting the mean firing rate of each of the three subpopulations over the sound stimulation and stimulation-free phases of our protocol. In the awake state, the sound specific population is boosted during sound stimulation whereas the ongoing assembly specific population responds more in the stimulation-free period. Under anesthesia, these modulations disappeared (Fig. 3G, S8).

To further validate our description of population activity in wakefulness, we simulated four different hypotheses about the relationship between the probability to be recruited in a soundevoked response and the probability to be recruited in an ongoing assembly. First, if we supposed that evoked and ongoing probabilities are independent and reshuffle all probabilities across cells, we generated a much broader distribution of ongoing and evoked probabilities across cells than actually observed (Fig. 3H) . If we supposed that ongoing and evoked probabilities are equal, we generated a narrower distribution of probabilities than observed (Fig. 3I). This model however resembled the distribution observed under anesthesia (Fig. 3F). We then supposed the existence of three neural populations: one with equal probability for ongoing and evoked assemblies, one with larger and one with smaller ongoing probability (Fig. 3K-L). This model much better fitted the distribution of observed probabilities provided that the proportions of neurons in the specific populations are around 15\% (Fig. 3L).

Our data show that sensory inputs and ongoing activity engages distinct neuronal assemblies in cortex during wakefulness, but that, during anesthesia, sensory-evoked and on-going assemblies become identical. This corroborates previous findings in anesthetized animals (2, 5). Our observation that the thalamic input is different for ongoing and sensory-driven activity, both in wakefulness and anesthesia, indicates that the merging of on-going and evoked assemblies is of cortical origin. The ability for the sensory cortex to form new, stimulus-specific neuronal assemblies that encode precise information about the sensory input could be a marker of wakefulness with respect to anesthesia. During anesthesia, although sensory input evokes responses, cortical assemblies lack stimulus specificity. Because sensory input just evokes patterns of spontaneous activity, one may assume that propagation of sensory information to other cortical areas will be limited, in agreement with findings in human anesthesia $(21,22)$. This may explain the loss of perception during anesthesia, and our results show that this loss already occurs in the primary sensory cortex. Our results are also compatible with the idea that in the awake cortex, the new assemblies formed by sensory inputs will propagate across the brain, but the mechanisms underlying this selective propagation are presently unknown, although previous models have proposed that asynchronous states may be the substrate of this selective propagation (23). 


\section{References and Notes}

1. A. Arieli, D. Shoham, R. Hildesheim, A. Grinvald, Coherent spatiotemporal patterns of ongoing activity revealed by real-time optical imaging coupled with single-unit recording in the cat visual cortex. J. Neurophysiol. 73, 2072-2093 (1995).

2. T. Kenet, D. Bibitchkov, M. Tsodyks, A. Grinvald, A. Arieli, Spontaneously emerging cortical representations of visual attributes. Nature. 425, 954-956 (2003).

3. J. E. Miller, I. Ayzenshtat, L. Carrillo-Reid, R. Yuste, Visual stimuli recruit intrinsically generated cortical ensembles. Proc Natl Acad Sci U A. 111, E4053-61 (2014).

4. S. Xu, W. Jiang, M. Poo, Y. Dan, Activity recall in a visual cortical ensemble. Nat. Neurosci. 15, 449-455 (2012).

5. A. Luczak, P. Bartho, K. D. Harris, Spontaneous events outline the realm of possible sensory responses in neocortical populations. Neuron. 62, 413-25 (2009).

6. J. Reimer, E. Froudarakis, C. R. Cadwell, D. Yatsenko, G. H. Denfield, A. S. Tolias, Pupil Fluctuations Track Fast Switching of Cortical States during Quiet Wakefulness. Neuron. 84, 355-362 (2014).

7. M. J. McGinley, M. Vinck, J. Reimer, R. Batista-Brito, E. Zagha, C. R. Cadwell, A. S. Tolias, J. A. Cardin, D. A. McCormick, Waking State: Rapid Variations Modulate Neural and Behavioral Responses. Neuron. 87, 1143-1161 (2015).

8. C. Stringer, M. Pachitariu, N. Steinmetz, C. B. Reddy, M. Carandini, K. D. Harris, Spontaneous behaviors drive multidimensional, brainwide activity. Science. 364 (2019), doi:10.1126/science.aav7893.

9. S. Musall, M. T. Kaufman, A. L. Juavinett, S. Gluf, A. K. Churchland, Single-trial neural dynamics are dominated by richly varied movements. Nat. Neurosci. 22, 1677-1686 (2019).

10. B. Molnár, P. Sere, S. Bordé, K. Koós, N. Zsigri, P. Horváth, M. L. Lörincz, Cell TypeSpecific Arousal-Dependent Modulation of Thalamic Activity in the Lateral Geniculate Nucleus. Cereb. Cortex Commun. 2, tgab020 (2021).

11. O. I. Rumyantsev, J. A. Lecoq, O. Hernandez, Y. Zhang, J. Savall, R. Chrapkiewicz, J. Li, H. Zeng, S. Ganguli, M. J. Schnitzer, Fundamental bounds on the fidelity of sensory cortical coding. Nature. 580, 100-105 (2020).

12. T. W. Chen, T. J. Wardill, Y. Sun, S. R. Pulver, S. L. Renninger, A. Baohan, E. R. Schreiter, R. A. Kerr, M. B. Orger, V. Jayaraman, L. L. Looger, K. Svoboda, D. S. Kim, Ultrasensitive fluorescent proteins for imaging neuronal activity. Nature. 499, 295-300 (2013).

13. B. Roland, T. Deneux, K. M. Franks, B. Bathellier, A. Fleischmann, Odor identity coding by distributed ensembles of neurons in the mouse olfactory cortex. Elife. 6 (2017), doi:10.7554/eLife.26337.

14. T. Deneux, A. Kaszas, G. Szalay, G. Katona, T. Lakner, A. Grinvald, B. Rozsa, I. Vanzetta, Accurate spike estimation from noisy calcium signals for ultrafast three-dimensional imaging of large neuronal populations in vivo. Nat Commun. 7, 12190 (2016).

15. B. Bathellier, L. Ushakova, S. Rumpel, Discrete neocortical dynamics predict behavioral categorization of sounds. Neuron. 76, 435-49 (2012).

16. M. R. DeWeese, A. M. Zador, Non-Gaussian membrane potential dynamics imply sparse, synchronous activity in auditory cortex. J Neurosci. 26, 12206-18 (2006).

17. J. Z. See, C. A. Atencio, V. S. Sohal, C. E. Schreiner, Coordinated neuronal ensembles in 
primary auditory cortical columns. eLife. 7, e35587 (2018).

18. P. H. C. Eilers, H. F. M. Boelens, Baseline correction with asymmetric least squares smoothing, (2005), (available at http://www.science.uva.nl/hboelens/publications/drapub/ Eilers_2005.pdf).

19. M. B. Pardi, J. Vogenstahl, T. Dalmay, T. Spanò, D.-L. Pu, L. B. Naumann, F. Kretschmer, H. Sprekeler, J. J. Letzkus, A thalamocortical top-down circuit for associative memory. Science. 370, 844-848 (2020).

20. S. A. Vasquez-Lopez, Y. Weissenberger, M. Lohse, P. Keating, A. J. King, J. C. Dahmen, Thalamic input to auditory cortex is locally heterogeneous but globally tonotopic. eLife. $\mathbf{6}$, e25141 (2017).

21. M. Massimini, F. Ferrarelli, R. Huber, S. K. Esser, H. Singh, G. Tononi, Breakdown of cortical effective connectivity during sleep. Science. 309, 2228-2232 (2005).

22. A. G. Casali, O. Gosseries, M. Rosanova, M. Boly, S. Sarasso, K. R. Casali, S. Casarotto, M.-A. Bruno, S. Laureys, G. Tononi, M. Massimini, A theoretically based index of consciousness independent of sensory processing and behavior. Sci. Transl. Med. 5, 198ra105 (2013).

23. Y. Zerlaut, A. Destexhe, Enhanced Responsiveness and Low-Level Awareness in Stochastic Network States. Neuron. 94, 1002-1009 (2017).

Acknowledgements: We thank Thomas Deneux, Guillaume Hucher and Aurélie Daret for their technical support, Melody Torao-Angosto for help with pilot experiments and numerous colleagues for comments on analyses and figures.

\section{Funding:}

European Community, Future and Emerging Technologies program (Human Brain Project, H2020-945539) (AD)

Paris-Saclay University "Initiatives de Recherches Stratégiques" - NeuroSaclay and Icode $(\mathrm{BB}, \mathrm{AD})$

Agence Nationale pour la Recherche 12-PDOC-0006, (BB), PARADOX (AD)

Region Ile de France - DIM Cerveau Pensée - MULTISENSE (BB)

Fondation pour 1'Audition, FPA IDA02 and APA 2016-03 (BB).

European Research Council, ERC CoG 770841 DEEPEN, (BB)

BB acknowledges the support of the Fondation pour l'Audition to the Institut de l'Audition. 


\section{Author contributions:}

Conceptualization: AF, AD, BB

Methodology: AF, AD, BB

Investigation: AF, BB

Visualization: AF

Funding acquisition: AD, BB

Project administration: AD, BB

Supervision: AD, BB

Writing - original draft: AF, AD, BB

Writing - review \& editing: $\mathrm{AF}, \mathrm{AD}, \mathrm{BB}$

Competing interests: The authors declare that they have no competing interest.

Data and materials availability: All data used in the analysis are available on Zenodo (10.5281/zenodo.5270513), code and materials are available upon request.

\section{Supplementary Materials}

Materials and Methods

Figs. S1 to S8 
A

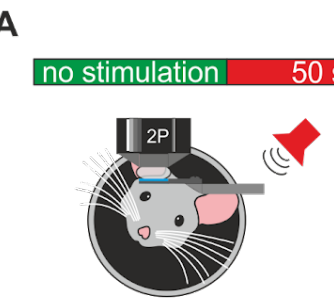

C

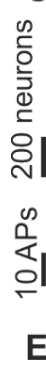

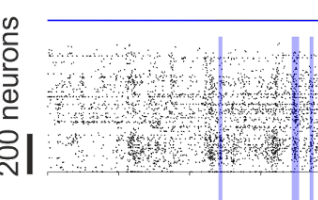

京
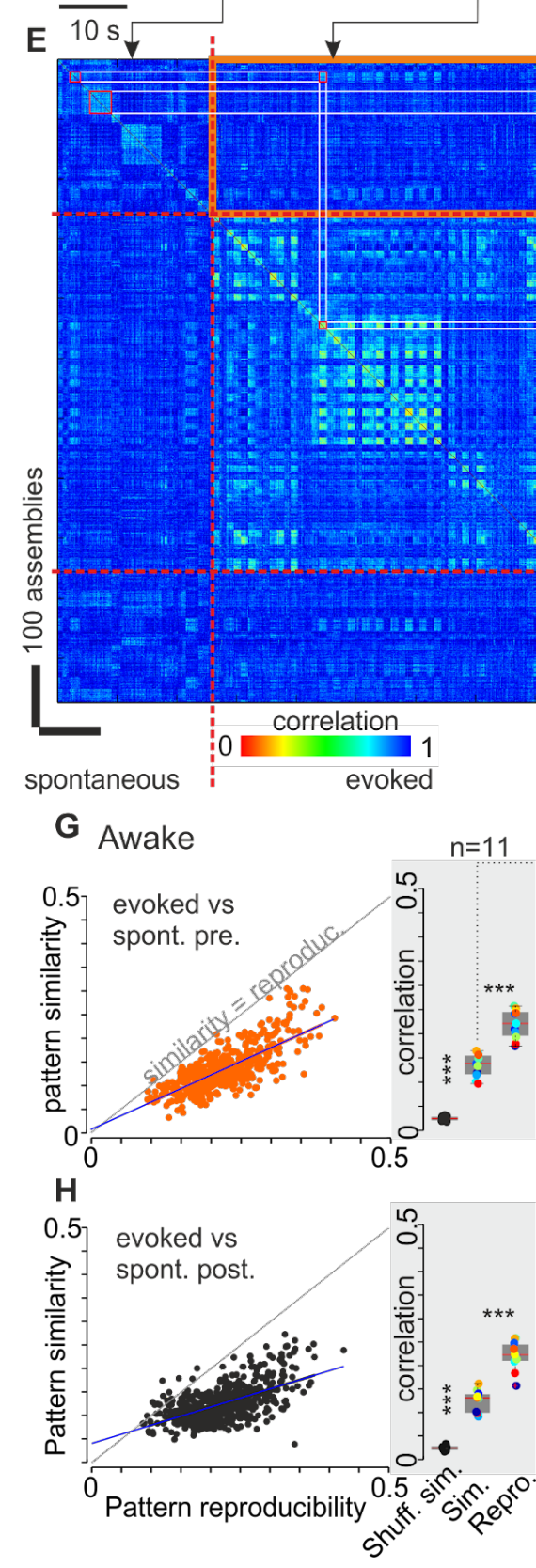

Awake

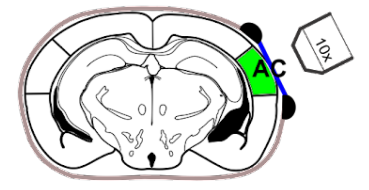

Sounds

$\|+1-1+\cdots+1+1+1+1+1-1$.

(n)

$500 \mathrm{~ms}$
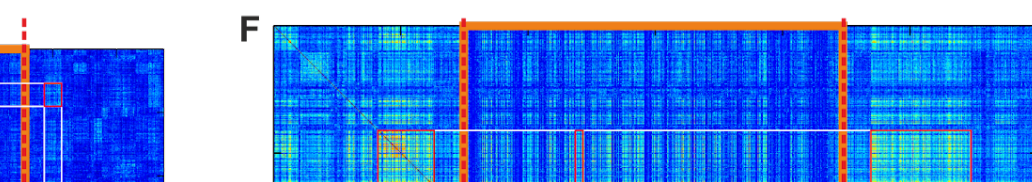
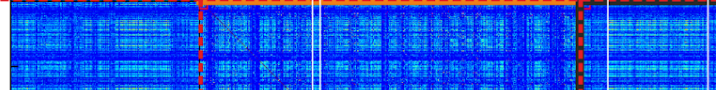

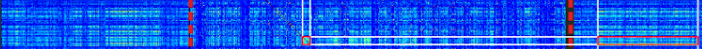

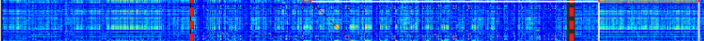

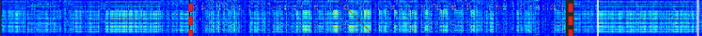

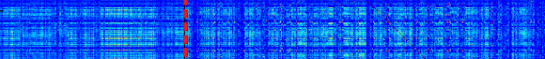

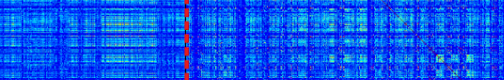

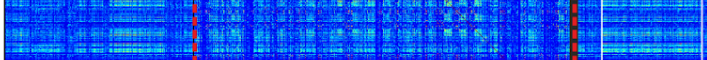

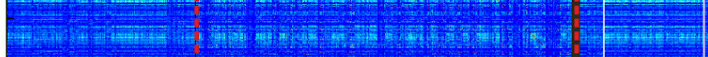

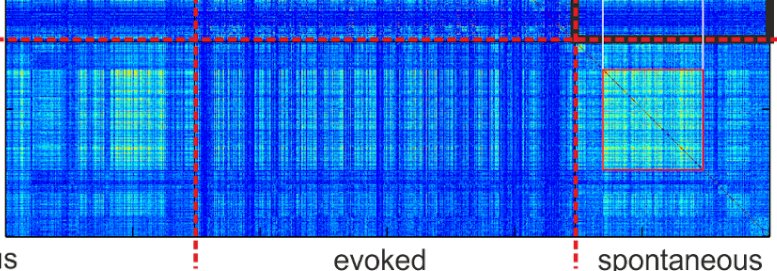

spontaneous

I Awake vs anesthesia

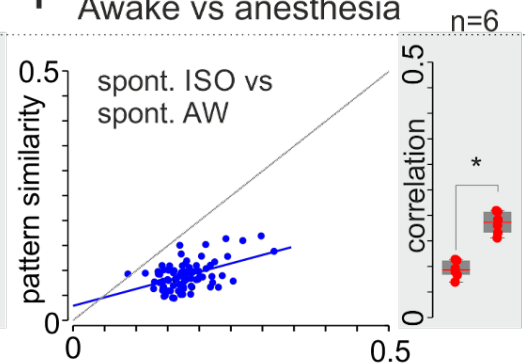

J

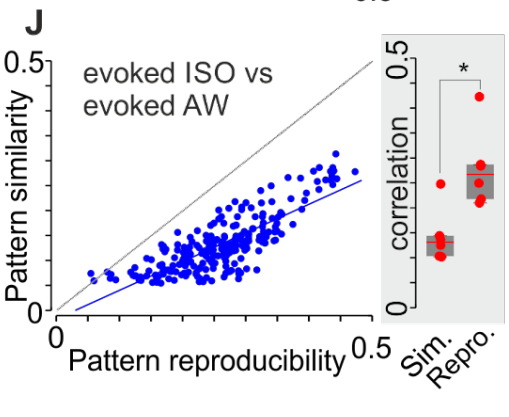

$\mathrm{K}$ Anesthesia

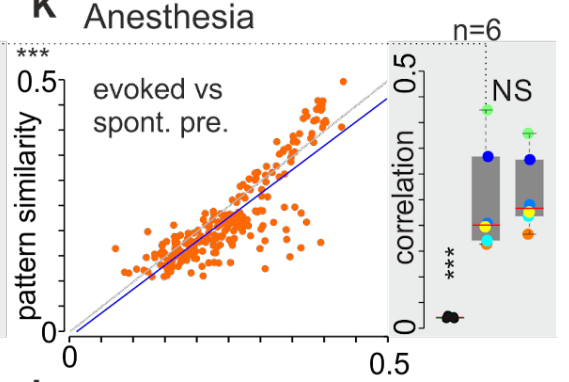

L

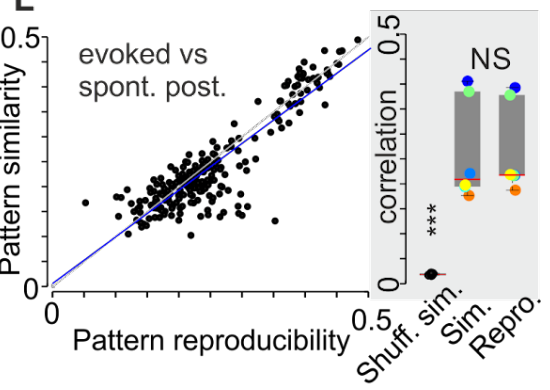


Fig 1. Ongoing and evoked population activity differ in the awake state but overlap under anesthesia. (A) Two-photon $\mathrm{Ca}^{2+}$ imaging at $30 \mathrm{~Hz}$ sampling rate of up to 1200 layer $2 / 3$ neurons expressing GCamp6s (AAV1 viral injection) in the awake head-fixed mouse. A $15 \mathrm{~min}$ sound stimulation was flanked by two $10 \mathrm{~min}$ long stimulation free periods. (B) The same recording protocol was applied under light isoflurane anesthesia (1.3\%). Right panel: spikes time estimates (red) were extracted from calcium fluorescence traces (blue) using the MLSpike algorithm. (C) Population raster plots and population firing rate (30ms bins) during no stimulation (left) and stimulation (right) periods. Vertical transparent bars highlight spontaneous (blue) and evoked (pink) population events detected as described in Fig. S1. (D). Same as in C for anesthesia. (E) For an example recording session, Pearson's correlation matrix between spontaneous assemblies sorted by hierarchical clustering and single trial sound response patterns (whether or not a population event was detected), sorted sound by sound (12 trials/sound). Lower correlation inside black and orange frames (similarity) compared to correlations along the diagonal (reproducibility) indicate that spontaneous and evoked patterns are different. (F) Correlation matrix under anesthesia for the same neuronal population as in E. Similar correlation in black and orange frames (similarity) and in the squares along the diagonal (reproducibility) indicate that spontaneous and evoked assemblies are highly similar. (G-L) Relation between reproducibility (abscissa) and similarity (ordinate) of sound-evoked and spontaneous patterns for all sounds and sessions. Statistics across sessions are given on the right-hand-side histograms (G: $\mathrm{p}=0.001, \mathrm{p}=0.001, \mathbf{H}: \mathrm{p}=0.001, \mathrm{p}=0.001$, Paired Wilcoxon Signed Rank Test, $\mathrm{n}=11$ mice). Spontaneous and evoked patterns can be considered dissimilar if their reproducibility is significantly larger than their similarity (grey: line for the equality, dark blue: data trend). A significant difference between spontaneous and evoked patterns is seen in awake mice (G-H), but not under anesthesia (K-L, p=0.44, $\mathrm{p}=0.56$, Paired Wilcoxon Signed Rank Test, $\mathrm{n}=6$ mice) where the similarity increased significantly (dashed line, G vs K, p=0.0003, Wilcoxon Ranksum test). Evoked and spontaneous population activity patterns under anesthesia are different from corresponding ones in the awake state $(\mathbf{I}-\mathbf{J}, \mathrm{p}=0.03, \mathrm{p}=0.03$ Paired Wilcoxon Signed Rank Test, $\mathrm{n}=6$ mice). 
Fig. 2
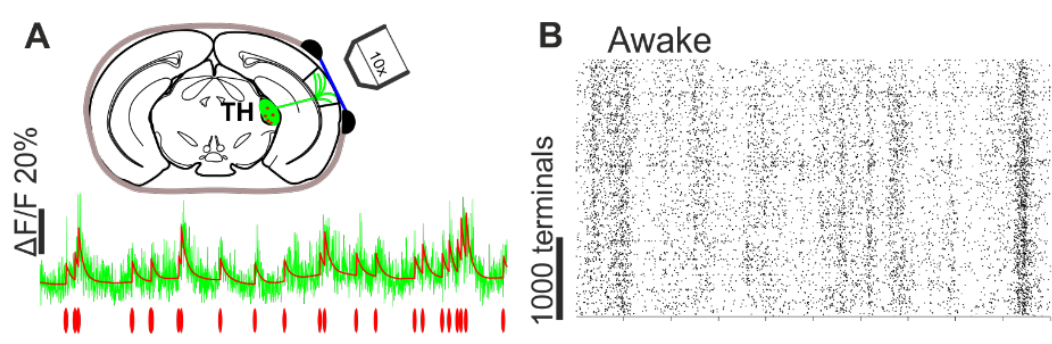

C Anesthesia

D

Awake

E

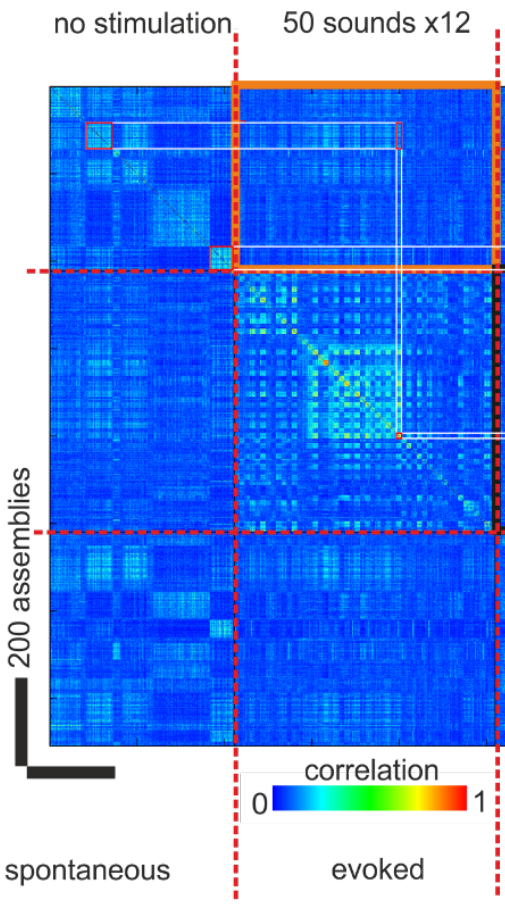

no stimulation

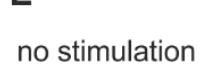

Anesthesia

50 sounds $\times 12$, no stimulation
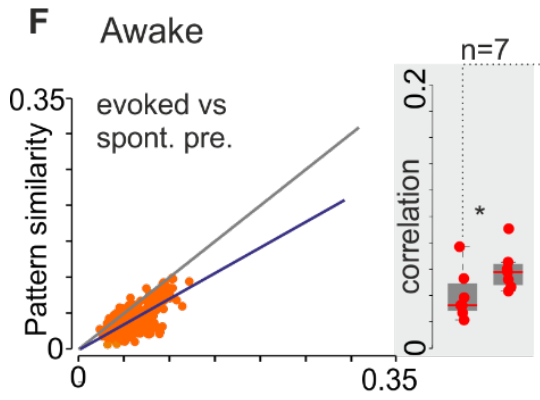

H Awake vs anesthesia

spontaneous
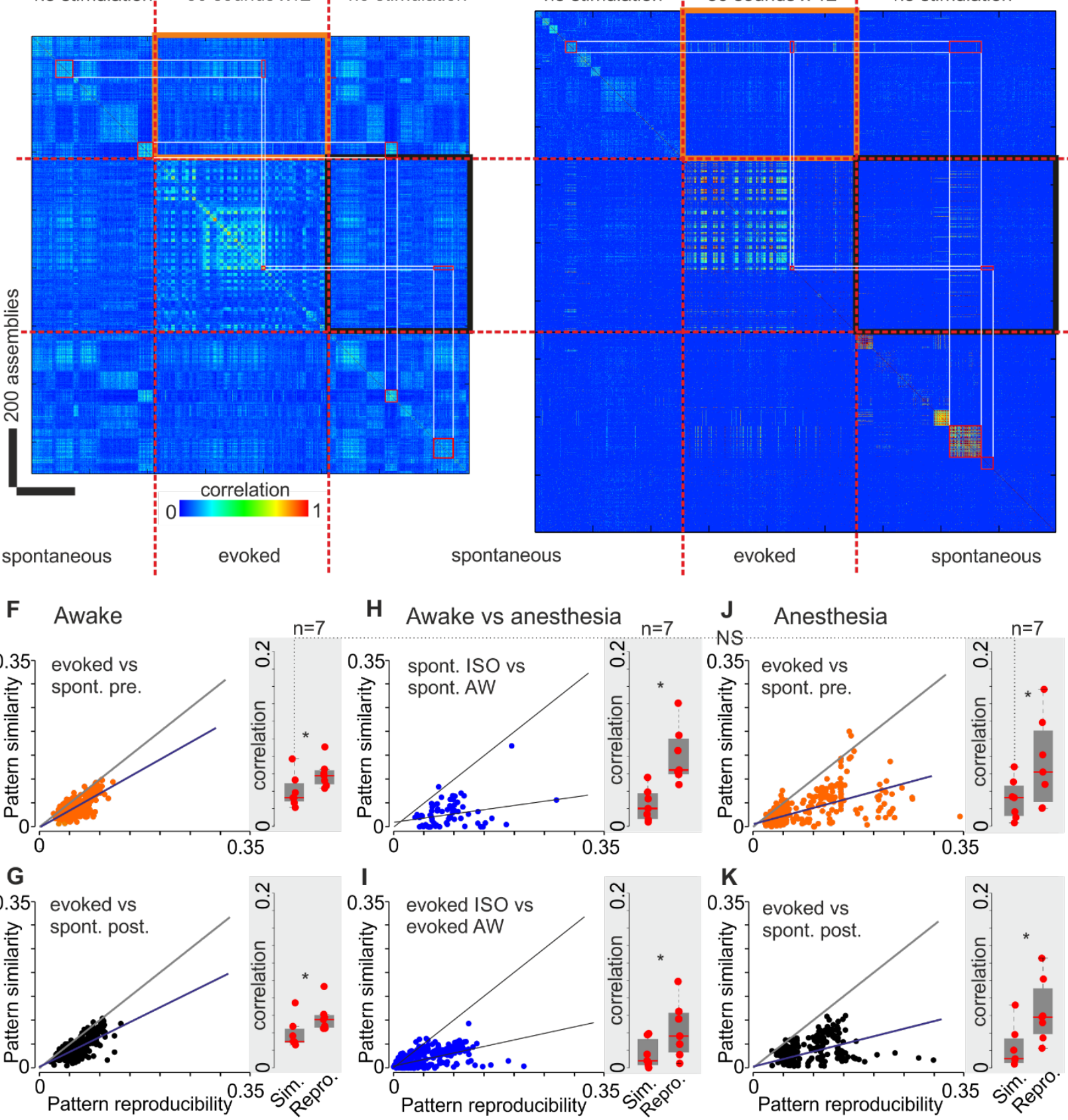
Fig 2. Auditory thalamus ongoing and evoked population activity patterns differ in both awake and anesthetized states. (A) Two-photon $\mathrm{Ca}^{2+}$ imaging at $30 \mathrm{~Hz}$ sampling rate of up to 4000 thalamo-cortical terminals expressing GCamp6s (AAV1 viral injection into the thalamus) in the AC layer 1 of awake head-fixed mouse. Below: example of thalamo-cortical terminal calcium trace (green) with spikes time estimates (red) using the MLSpike algorithm. (B) Population raster plot in awake state where each line represents spontaneous spiking pattern of a thalamo-cortical terminal. (C) Same under light isoflurane anesthesia (1.3\%). (D) For an example recording session, Pearson's correlation matrix between spontaneous assemblies of thalamo-cortical terminals sorted by hierarchical clustering and single trial sound response patterns (whether or not a population event was detected), sorted sound by sound (12 trials/ sound) (E) Same as D under anesthesia. Lower correlation inside black and orange frames (similarity) compared to correlations along the diagonal (reproducibility) indicate that spontaneous and evoked patterns are different. (F-K) Relation between reproducibility (abscissa) and similarity (ordinate) of sound-evoked and spontaneous patterns for all sounds and sessions. Statistics across sessions are given on the right-hand-side histograms (F-K: $p=0.016$, Paired Wilcoxon Signed Rank Test, $\mathrm{n}=7$ mice). Spontaneous and evoked patterns are dissimilar in both awake (F-G) and anesthetized states (J-K) (grey: line for the equality, dark blue: data trend) and neither of them keep similitude passing from one state to another $(\mathbf{H}-\mathbf{J})$. 
A $n=11$ mice 6310 neurons
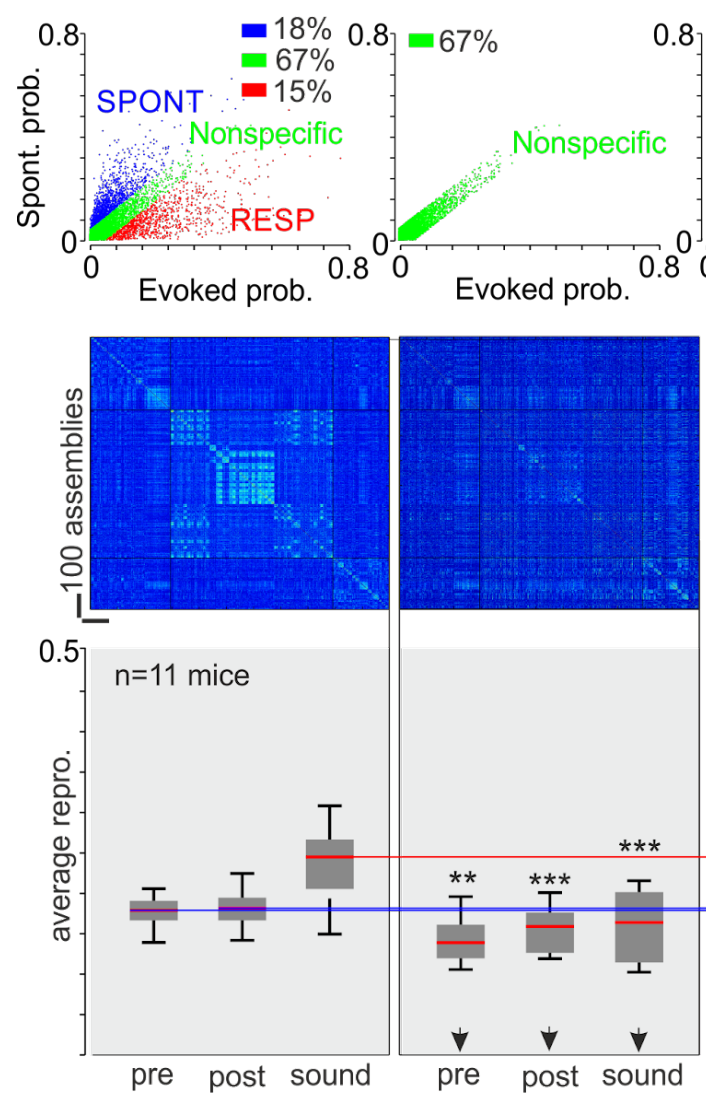

$\mathbf{F}$
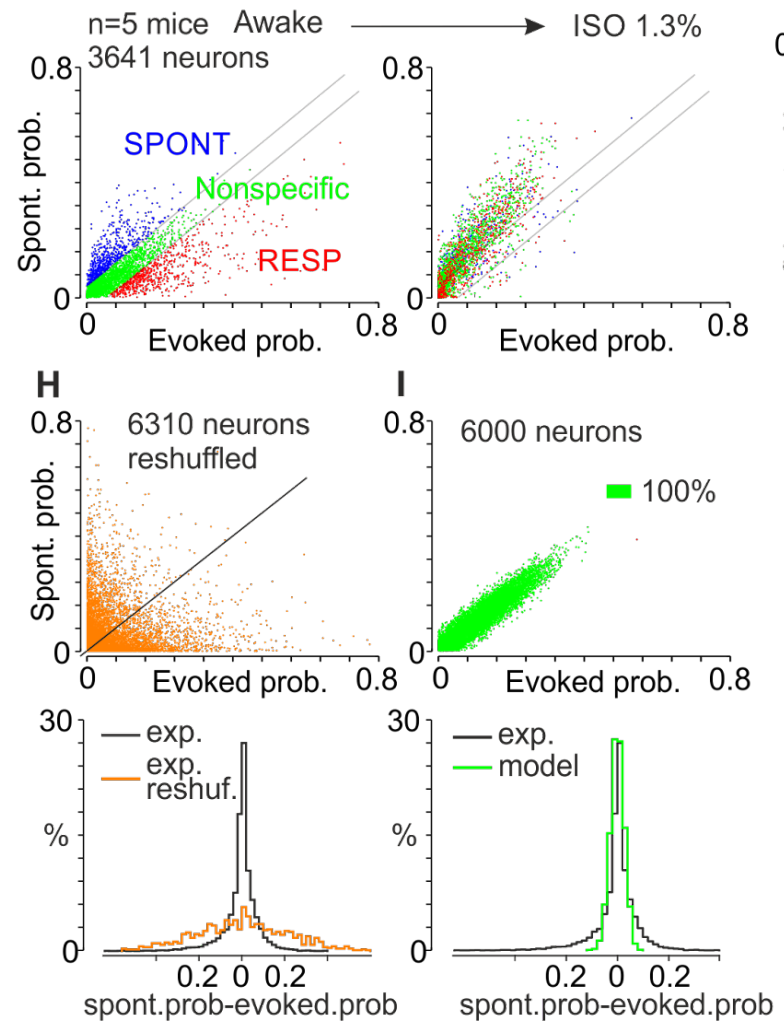

C

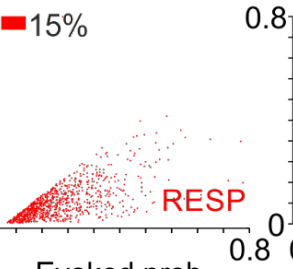

D

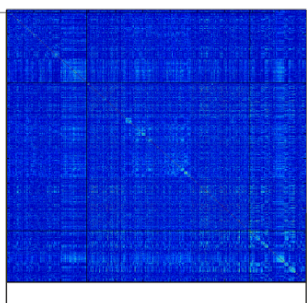

Evoked prob.

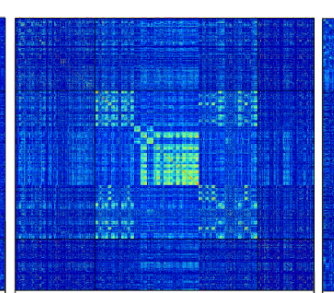

Evoked prob.

E
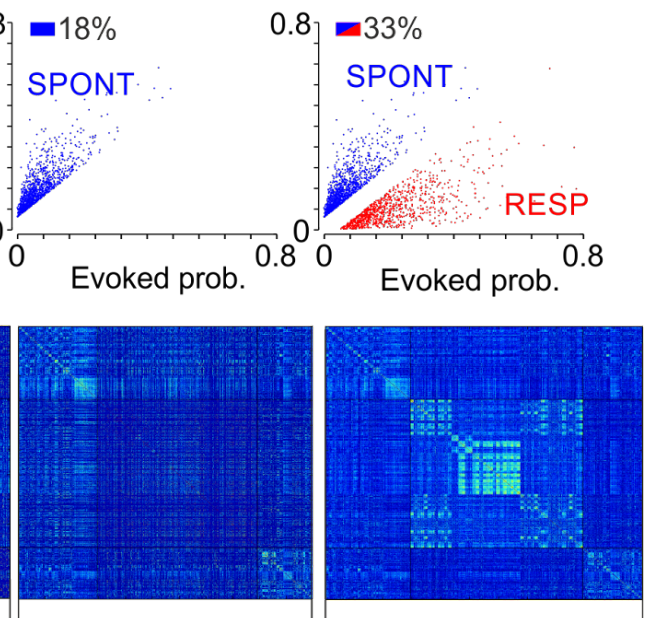

G

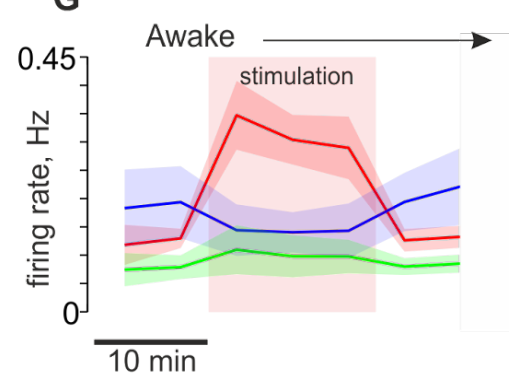

ISO $1.3 \%$
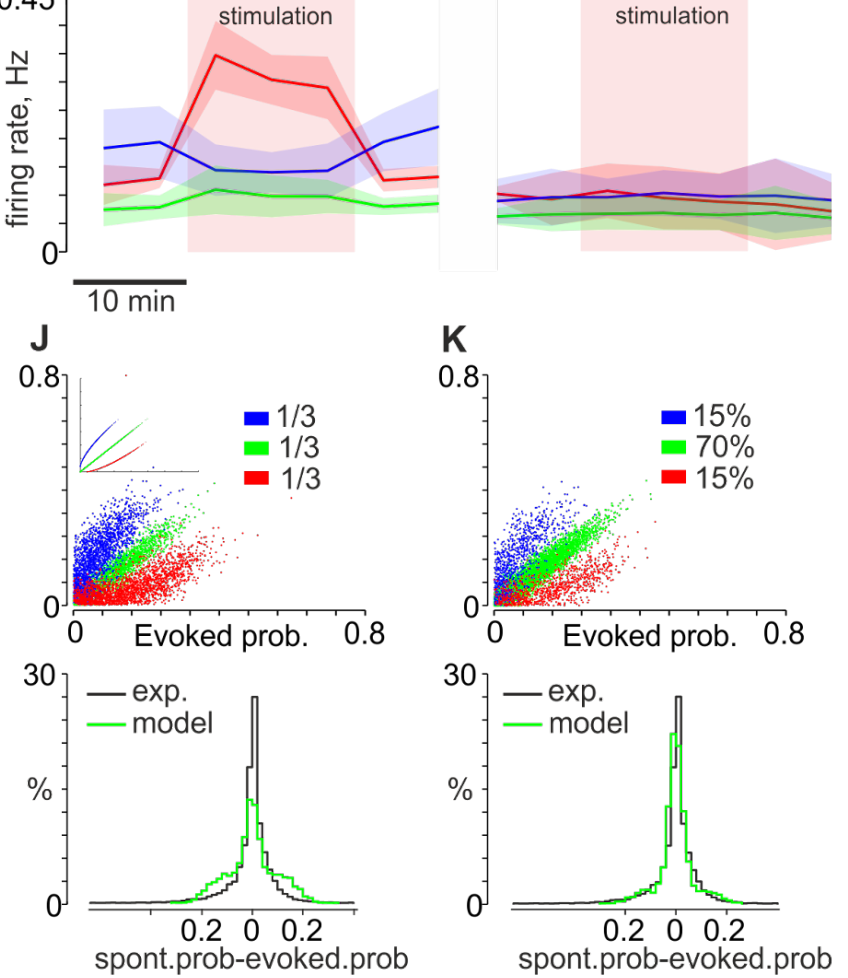
Fig 3. Specificity of evoked patterns rely on a distinct population of neurons. (A) Top: Plot of the probability to respond to any sound versus the probability to be recruited in an on-going event for 6310 neurons over 11 mice. The color code indicates whether the neuron prefers ongoing (blue) or evoked (red) events or nonspecifically participates in both types of events (boundaries: +/- 1 mean absolute deviation of the probability difference). Middle: For a sample session, Pearson's correlation matrix computed with all available neurons. Bottom: Average sound and spontaneous event cluster reproducibility (B-E: $p=0.002 / 0.001 / 0.001$ $\mathrm{p}=0.001 / 0.02 / 0.001, \mathrm{p}=0.001 / 0.001 / 0.001, \mathrm{p}=0.001 / 0.001 / 0.001$ Paired Wilcoxon Signed Rank Test, $\mathrm{n}=11$ mice). (B). Same as A, but correlation matrix and reproducibility are calculated with nonspecific neurons only (67\%). (C). Same as A, but for sound responsive neurons only (15\%). (D). Same as A, but only for neurons preferring on-going events (18\%). (E). Same as D but for all except nonspecific neurons (33\%). (F). Plot of the probability to respond to any sound versus the probability to be recruited in an on-going event for 3641 neurons over 5 mice in awake (left) and under anesthesia (right). The color code is defined as in A for the awake state. Under anesthesia, the three color-coded populations converge to a single group with strongly correlated probability of activation in spontaneous and evoked events. (G). Time course of the mean firing rate profiles for each of the 3 groups of neurons in awake (left) and under anesthesia (right). Under anesthesia, specificity to sound and ongoing activity disappears. (H) Top: same as A, but probabilities are reshuffled along each axis to show the expected probability distribution for independent participations to on-going and evoked events. Bottom: corresponding distribution of probabilities difference (on-going - evoked, 6310 neurons, over 11 mice). Experimental (black), reshuffled (orange). (I) Probability distributions for 6000 surrogate neurons with equal participation probabilities to on-going and evoked events. (J) Probability distributions for 6000 surrogate neurons divided in three groups of equal size: two groups with a larger (resp. smaller) participation probability for on-going events, one group with equal probability. (K) Same as J, but for different group sizes. 